\section{Recent developments in HIV treatment and their dissemination in poor countries}

\author{
Osman Ebrahim, ${ }^{1}$ \\ Ahmad Haeri Mazanderani ${ }^{2}$ \\ 'Department of Medical Microbiology, \\ Faculty of Health Sciences, University \\ of Pretoria; '2Department of Medical \\ Virology, Faculty of Health Sciences, \\ University of Pretoria and National \\ Health Laboratory Service, Tshwane \\ Academic Division, South Africa
}

\section{Abstract}

As the world enters the fourth decade of the HIV/AIDS epidemic a number of new drugs have been developed that address current challenges with antiretroviral therapy (ART), such as pill burden, toxicity and drug-resistance. These new agents have not only been developed from established drug-classes, namely nucleoside reverse transcriptase inhibitors (NRTIs), non-nucleoside reverse transcriptase inhibitors (NNRTIs) and protease inhibitors (PIs), but also include innovative ways of suppressing viral replication. Intergrase inhibitors and chemokine receptor blockers have been developed which, combined with NRTIs, NNRTIs and PIs, comprise highly active antiretroviral therapy regimens able to tackle all aspects of the HIV life cycle with minimal toxicity. Furthermore, the ability of pharmaceutical companies to formulate these powerful drugs into fixed-dose combinations provides exciting new strategies for reducing pill burden, thus ensuring adherence and limiting the emergence of drug-resistance. The enthusiasm with which these new drugs have been received has, however, been tempered by the reality of limited access in the developing world, further highlighting the disparity between rich and poor countries in the fight against HIV/ AIDS. Access to these treatments in low- and middle-income countries will require the necessary political will, regulatory approval, affordability of drugs, as well as efficient procurement and supply management strategies. The priority of developing countries remains increased scale up of ART, but there is also a need to acquire new drugs in order to tackle toxicity and drug-resistance, both of which threaten the sustainability of such programmes. Thankfully, the vast majority of patients receiving ART in the developing world are still on first-line regimens, thus allowing time for newer agents to be made available as part of third-line treatment option. However, there is no room for complacency - the develop- ing world needs access to new HIV treatments, an AIDS-free generation depends upon it.

\section{Introduction}

In June 2011, as the world entered the fourth decade of the HIV/AIDS epidemic, UN Member States unanimously endorsed a new set of Global AIDS Response targets to be achieved by 2015 . These included eliminating new infections amongst children, substantially reducing the number of AIDS-related maternal deaths, and halving the sexual transmission of HIV. ${ }^{1}$ Despite the absence of an effective vaccine and an incidence of 2.5 million new infections last year, $70 \%$ of whom reside in subSaharan Africa, there has been renewed hope that the epidemic can be controlled. ${ }^{2}$ The optimism is partly on account of evidence which suggests antiretroviral therapy (ART) can effectively prevent the transmission of HIV, ${ }^{3}$ and data demonstrating a decline in incidence and death rates in some of the most afflicted countries of the world. ${ }^{2}$ HIV treatment is estimated to have saved 14 million life years since 1995 in low- and middle-income countries and genuine efforts are underway to scale up rollout programmes with a target of having 15 million HIV-infected people on treatment by 2015. ${ }^{1,2}$ Amid this renewed emphasis on treatment, we look at recent developments in the field of antiretroviral therapy, and in particular their role and dissemination in the developing world.

Currently 8 million people access ART globally, with $54 \%$ of people who are eligible for treatment in the developing world receiving ART. ${ }^{2}$ The majority of this treatment is made available through donor-sponsored roll-out programmes which have adopted a publichealth approach as advocated by the World Health Organisation (WHO). In contrast to individualized specialist physician management, these programmes have been designed for low- and middle-income countries as a means of providing greater access to ART whilst taking into consideration the limited resources and expertise available. ${ }^{4}$ By employing standardized and simplified treatment protocols real progress has been made in scalingup HIV/AIDS services in the developing world. ${ }^{2}$ However, a successful ART programme not only depends on the accessibility of drugs, but also continued adherence to medicines that remain effective. Apart from societal factors, and in particular community leadership, which is increasingly being appreciated as having a central role in the sustainability of HIV treatment programmes, ${ }^{2}$ the antiretrovirals (ARVs) themselves critically influence the distribution, adherence and effectiveness of HIV treatment.
Correspondence: Osman Ebrahim, Department of Medical Microbiology, Faculty of Health Sciences, University of Pretoria, P0 Box 1073, Houghton 2041, Johannesburg, South Africa.

Tel. +27.11.6479142 - Fax: +27.11.6428921

E-mail: osman.ebrahim@telkomsa.net

Key words: HIV/AIDS, antiretroviral therapy, developing countries.

Conflict of interest: the authors declare no conflict of interests.

Received for publication: 19 February 2013. Accepted for publication: 19 February 2013.

This work is licensed under a Creative Commons Attribution NonCommercial 3.0 License (CC BYNC 3.0).

(C) Copyright O. Ebrahim and A.H. Mazanderani, 2013

Licensee PAGEPress, Italy

Infectious Disease Reports 2013; 5:sle2

doi:10.4081/idr.2013.s1.e2

The WHO currently recommends one of two treatment regimens - a first-line regimen (an NNRTI plus two NRTIs, one of which should be AZT or TDF) for initiating treatment in all those who are HIV positive with CD4+ T cell counts of less than 350 cells l (and/or have stage III-IV disease), and a second-line regimen (a ritonavir boosted PI plus two NRTIs, one of which should be AZT or TDF depending on first-line choice. ATV/r or LPV/r are the preferred PIs) in the event of first-line treatment failure. ${ }^{5}$ Both regimens contain a cocktail of three drugs which were developed over a decade ago. Subsequently, new HIV treatments have emerged within the global ART landscape which promise to facilitate scaling up distribution of ART by lowering costs and assisting supply management; improving adherence on account of better side-effect profiles, easier to administer formulations and reduced pill-burden; and enhancing efficacy by providing the possibility of a third-line regimen in the event of drug-resistance. These new medicines include fixed-dose combinations (FDCs), better drug formulations, new drugs belonging to established classes, such as second-generation NNRTIs and PIs, and agents from whole new drug-classes, which include the integrase inhibitors and fusion inhibitors. Appreciating the need to improve the efficiency and effectiveness of HIV treatment programmes, the WHO and the Joint United Nations Programme on HIV/AIDS (UNAIDS) launched Treatment 2.0 in July 2010 , a strategy to further simplify treatment regimens to support the scale up of ART by promoting the use of new drugs. ${ }^{6}$ The interconnectivity between distribution, adherence and efficacy of antiretroviral therapy, and the potential impact of new developments in 
HIV treatments on ART roll-out programmes in the developing world is the focus of this review. We will first discuss new drug innovations, including FDCs, new agents with reduced side-effects, better formulations, and drugs that can be used to address the increasing threat of drug-resistance. Thereafter we briefly discuss the distribution of new HIV treatments in the developing world, and the shortcoming thereof, with a focus on South Africa.

\section{New drug developments}

There are currently 27 FDA approved ARVs, and more than 15 drugs and combinations in the development pipeline. ${ }^{6}$ Each agent targets a specific point in the HIV life-cycle, with a suppressive ARV regimen having the net effect of preventing viral replication. Drug classes include the older more established nucleoside and nucleotide reverse transcriptase inhibitors (NRTIs and NtRTIs), non-nucleoside reverse transcriptase inhibitors (NNRTIs) and the protease inhibitors (PIs). Two new classes have recently been added to the armamentarium, these being the integrase inhibitors and entry inhibitors (including CCR5 coreceptor antagonists and fusion inhibitors). For a list of new developments, both approved and in the pipeline, please refer to Table $1 .^{7}$

\section{Fixed dose combinations}

The WHO suggests regimens that are easy to administer, such as those that involve fewer pills with simple dosing schedules, as a way to ensure or improve patient adherence. ${ }^{6}$ FDCs have been endorsed as a solution to the numerous pills and dosing schedules involved in taking Highly Active Antiretroviral Treatment (HAART), with some combinations containing a fully-suppressive HAART regimen that requires a single daily dose and doesn't need refrigeration. A number of such FDCs have been developed for first-line therapy but have been slow to penetrate treatment programmes in the developing world. HAART regimens in a single tablet (TDF+FTC+EFV, marketed as atripla) have been available in the US since 2006 and promise to be cost effective treatment options. South Africa, which has the largest antiretroviral programme in the world, will finally have such a combination starting in 2013 with the South African department of health reporting to have saved R2.2 billion on its latest tender. ${ }^{8}$ Apart from improving treatment adherence FDCs are also considered to assist prescribing and aid procurement and supply management - important challenges facing roll-out programmes in the developing world.

FDCs are, of course, not without complications. No single regimen can safely be used for all patients and standardising treatment approaches to accommodate drug toxicity is challenging. ${ }^{4}$ The $\mathrm{WHO}$ has in the past used an FDC containing d4T+lamivudine+nevirapine as first-line therapy which caused considerable morbidity, the toxicity of $\mathrm{d} 4 \mathrm{~T}$ and nevirapine being well documented. ${ }^{9}$

Exciting new FDCs include Eviplera and Complera, which contains a cocktail of tenofovir, emtricitabine, and rilpivarine (an FDA approved second generation NNRTI), and Quad, a formulation of tenofovir, emtricitabine, elvitegravir and cobicistat. Quad is unique on account of it including a secondgeneration integrase inhibitor (elvitegravir) and the pharmacoenhancer cobicistat designed specifically as a pharmacokinetic booster. Eviplera has been found to be as effective as atripla with the added advantage of not containing efavirenz thus avoiding neuropsychiatric side effects, ${ }^{10}$ whereas Quad promises to be an attractive FDC which could potentially be used as a second- or third-line regimen that would avoid the neuropsychiatric symptoms of efavirenz and skin-rashes associated with other NNRTIs, and the metabolic side-effects commonly experienced with protease inhibitors. $^{11}$

A number of additional FDCs are in development including those based on cobicistat boosted darunavir, and the third integrase inhibitor, dolutegravir, both combined with two NtRTIs. ${ }^{7}$

\section{New drugs with good safety profile}

HIV co-infection with tuberculosis, viral hepatitis and other opportunistic infections is common, especially in the developing world. Simultaneous treatment of co-infections and HIV is problematic not only because of the high pill-burden but also due to drug-drug interactions, overlapping toxicities, and paradoxical reactions. This can potentially affect drug adherence and lead to treatment failure.

Stavudine (d4T), zidovudine (AZT) and didanosine (ddI) are still widely used in developing countries as an NRTI backbone in the first-line regimen. ${ }^{12}$ Toxicities from these medicines in the form of lipoatrophy, increased skin pigmentation, peripheral neuropathy and lactic acidosis are well documented. Tenofovir (TDF), an NtRTI, is in comparison well tolerated on account of having a low affinity for mitochondrial polymerase and can actually improve lipoatrophy and dyslipidaemia. ${ }^{13}$ Another advantage is its efficacy against hepatitis B which, along with lamivudine, make it an additionally attractive first-line option in areas with a high prevalence of hepatitis B, such as is in sub-Saharan Africa, China and SouthEast Asia. Tenofovir is of course not a new drug, having been approved by the FDA in 2001. However, it was very slow to be approved by regulatory agencies in the developing world, an important bottle-neck that we shall consider later in more detail, and it has only recently replaced d4T, AZT and ddI as a first-line agent in WHO guidelines. ${ }^{5}$

Tenfovir is, however, not without sideeffects, which include nephrotoxicity and bone demineralization. The prodrug of tenofovir, GS-7340, which requires a much reduced dosage may alleviate some of these problems. Newer NNRTIs and PIs also boast reduced side-effects, such as atazanavir and darunavir. A new heat-stable formulation of atazanavir/ritonavir is now available, and has the additional benefit of only requiring one pill taken daily - a much more favourable option compared with the twice daily dosing of two pills of heat-stable lopinavir-ritonavir. Furthermore, integrase inhibitors are unlikely to produce severe side effects as the enzyme integrase is not normally present in human cells.

\section{New formulations}

Recent developments include FDA approved oral suspension of darunavir for children older than 3 years of age; chewable raltegravir tablets for children 2-18 years old; oral powder and tablets of tenofovir for children 2-18 years old; and etravirine tablets for children 6-18 years old. ${ }^{11}$ However, genuine advancement in new-drugs designed for treating children with HIV is lacking and further research on appropriate drug regimens in paediatric and adolescent populations is critically needed. ${ }^{14}$

\section{New drugs and resistance}

Invariably, increased access to treatment brings new challenges, including the looming spectre of drug-resistance. A recent systematic review of the literature suggests that $60 \%$ of people failing NNRTI-based first line therapy in low- and middle-income countries have drug resistance. ${ }^{15}$ Drug resistance has been detected in $80 \%$ of South African patients who experienced failure of a first-line HAART regimen, with dual-class drug-resistant virus present in $64.3 \%$ of participants. ${ }^{16}$ Drug resistance can also be transmitted, with data from WHO surveys suggesting that greater coverage of antiretroviral therapy is associated with a higher 
Table 1. HIV treatment pipeline 2003-2012.

\begin{tabular}{|c|c|c|c|c|c|}
\hline Class & Drug name & Generic name & Brand name & Sponsor & Status \\
\hline NRTI & FTC & Emtricitabine & Emtriva & Triangle/Gilead & Approved (2003) \\
\hline NRTI & AG1549 & Capravirine & & Agouron/Pfizer & Discontinued (2005) \\
\hline NRTI & DAPD & Amdoxovir & & Gilead/Emory/RFS Pharma & Discontinued (2010) \\
\hline NRTI & MIV-310, FLT & Alovudine & & Boehringer Ingelheim/Medivir/Beijing Mefuvir & To Mefuvir (2008) \\
\hline NRTI & ACH-126443 & Elvucitabine & & Achillion & Phase II (2007) \\
\hline NRTI & D-d4T, DPC-817 & Reverset & & Pharmasset/Incyte & Discontinued (2006) \\
\hline NRTI & SPD-754, AVX-754, ATC & Apricitabine & & Shire BioChem/Avexa & Discontinued (2010) \\
\hline NRTI & & Racivir & & Pharmasset & Discontinued (2008) \\
\hline NRTI & 4'-Ed4T, OBP-601 (ex festinavir) & BMS-986001 & & Bristol-Myers Squibb & Phase II (2012) \\
\hline NRTI & CMX-157 & & & Chimerix & Phase I (2012) \\
\hline NtRTI & GS-7340, PMPA & & & Gilead & Phase II (2012) \\
\hline NNRTI & TMC-125 & Etravirine & Intelence & Janssen (ex Tibotec) & Approved (2008) \\
\hline NNRTI & & Calanolide A & & Advanced Life Sciences/ Sarawak MediChem & Phase II (2005) \\
\hline NNRTI & DPC-083, AI-183 & & & Bristol-Myers Squibb & Discontinued (2004) \\
\hline NNRTI & TMC-278 & Rilpivirine & Edurant & Janssen (ex Tibotec) & Approved (2011) \\
\hline NNRTI & BILR-355/r BS & & & Boehringer Ingelheim & Discontinued (2008) \\
\hline NNRTI & UK-453061 & Lersivirine & & Pfizer & Phase II (2012) \\
\hline NNRTI & & & Viramune XR & Boehringer Ingelheim & Approved (2011) \\
\hline $\begin{array}{l}\text { NNRTI } \\
\text { injectable }\end{array}$ & & Rilpivirine-LA & & Janssen (ex Tibotec) & Phase I (2012) \\
\hline PI & & Atazanavir & Reyataz & Bristol-Myers Squibb & Approved (2003) \\
\hline PI & VX-175, GW-433908 & Fosamprenavir & Lexiva & Vertex/GlaxoSmithKline & Approved (2003) \\
\hline PI & & Tipranavir & Aptivus & Boehringer Ingelheim & Approved (2005) \\
\hline PI & TMC-114 & Darunavir & Prezista & Janssen (ex Tibotec) & Approved (2006) \\
\hline PI & GSK-640385 & Brecanavir & & GlaxoSmithKline & Discontinued (2007) \\
\hline PI & PPL-100 & & 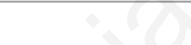 & Ambrillia/Merck & Discontinued (2008) \\
\hline FI & $\mathrm{T}-20$ & Enfuvirtide & Fuzeon & Trimeris/Hoffmann-La Roche & Approved (2003) \\
\hline CCR5RI & SCH-C, SCH-351125 & & 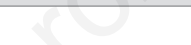 & Schering-Plough & Discontinued (2004) \\
\hline CCR5RI & UK-427857 & Maraviroc & Selzentry & Pfizer & Approved (2007) \\
\hline CCR5RI & SCD-D, SCH-417 & Vicriviroc & Whan & Schering-Plough & Discontinued (2010) \\
\hline CCR5RI/2RI & TAK-652, TBR-652 & Cenicriviroc & & Takeda/Tobira & Phase II (2012) \\
\hline InI & MK-0518 & Raltegravir & Isentress & Merck & Approved (2007) \\
\hline InI & GS-9137, JTK-303 & Elvitegravir & & Gilead & Submitted (2012) \\
\hline InI & S/GSK-1349572 & Dolutegravir & & GlaxoSmithKline/Shionogi/NiiV & Phase III (2012) \\
\hline InI & GSK-1265744 & & & GlaxoSmithKline/Shionogi & Phase II (2012) \\
\hline InI & GSK-1265744 (LA) & Long-acting GSK-1265744 & & GlaxoSmithKline/Shionogi & Phase I (2012) \\
\hline Anti-CD4 Mab & TNX-355, Hu5A8 & Ibalizumab & & Tanox/Biogen Idec/TaiMed & Phase II (2011) \\
\hline $\mathrm{AI}$ & PR0-542 & $x^{2}+x^{2}$ & & Progenics & Discontinued (2004) \\
\hline Al & PA-457, MPC-4326 & Bevirimat & & Panacos/Vitex/Myriad & Discontinued (2011) \\
\hline $\mathrm{AI}$ & PR0-140 & & & Progenics & Discontinued (2011) \\
\hline Al (gpl20) & BMS-663068 & & & Bristol-Myers Squibb & Phase II (2012) \\
\hline PK booster & GS-9350 & Cobicistat & & Gilead & Submitted (2012) \\
\hline PK booster & SPI-251 & & & Sequoia & Discontinued (2011) \\
\hline PK booster & CTP-518 & & & GlaxoSmithKline & On hold (2012) \\
\hline FDC & $\mathrm{ABC} / 3 \mathrm{TC}$ & Zidovudine/lamivudine & Epzicom & GlaxoSmithKline & Approved (2003) \\
\hline $\mathrm{FDC}$ & FTC/TDF & Emtricitabine/tenofovir & Truvada & Gilead & Approved (2004) \\
\hline $\mathrm{FDC}$ & $\mathrm{EFV/FTC/TDF}$ & $\begin{array}{l}\text { Efavirenz/emtricitabine/ } \\
\text { tenofovir }\end{array}$ & Atripla & $\begin{array}{l}\text { Bristol-Myers } \\
\text { Squibb/Gilead }\end{array}$ & Approved (2006) \\
\hline FDC & RLV/FTC/TDF & $\begin{array}{l}\text { Rilpivirine/emtricabine/ } \\
\text { tenofovir }\end{array}$ & Complera/Eviplera & Janssen (ex Tibotec)/Gilead & Approved (2011) \\
\hline $\mathrm{FDC}$ & EVG/COBI/FTC/TDF & $\begin{array}{l}\text { Elvitegravir/cobicistat/ } \\
\text { emtricitabine/tenofovir }\end{array}$ & Quad & Gilead & Submitted (2012) \\
\hline FDC & & $\begin{array}{l}\text { Elvitegravir/cobicistat/ } \\
\text { emtricitabine/GS-7340 }\end{array}$ & & Gilead & Phase II (2012) \\
\hline FDC & & Darunavir/cobicistat/emtricitabine & 7340 & Janssen (ex Tibotec)/Gilead & Phase II (2012) \\
\hline FDC & & Dolutegravir/abacavir/lamivudine & 72-Trii & ViIV & Phase III (2012) \\
\hline
\end{tabular}

Adapted from Clayden et al., 2012 with permission. ${ }^{7}$ NRTI, nucleoside reverse transcriptase inhibitor; NtRTI, nucleotide reverse transcriptase inhibitor; NNRTI, non-nucleoside reverese transcriptase inhibitor; PI, protease inhibitor; FI, fusion inhibitor; CCR5RI, CCR5 receptor inhibitor; CCR2RI, CCR2 receptor inhibitor; InI, integrase inhibitor; AI, attachment inhibitor; MI, maturation inhibitor; PK booster, pharmacokinetic booster; FDC, fixed dose combination. 
prevalence of transmitted drug resistance, particularly to NNRTIs. ${ }^{15} 8.6 \%$ of newly HIV-1 diagnosed young adults in Uganda have been found to have transmitted drug-resistance, compared with $0 \%$ in a study conducted four years earlier. ${ }^{17}$

The proportion of patients on a second-line regimen in resource-limited settings is currently estimated to be between $1-5 \% .{ }^{18}$ As scale up of ART continues and access to viral load monitoring improves, demand for second-line treatment will increase. Similarly, patients will increasingly need third-line regimens, as the inevitable eventuality of virological failure on second-line treatment arises. A number of new drugs have been approved to address these needs, including second generation NNRTIs, PIs, as well as new classes of drugs such as integrase inhibitors, CCR5 (chemokine receptor) antagonists and fusion inhibitors.

Darunavir, a new non-peptidic protease inhibitor, has demonstrated impressive potency in the presence of PI resistant mutants, hence its importance in treating therapy-experienced patients..$^{19}$ Etravirine, a second-generation NNRTI, was approved in 2008 for ARTexperienced adult patients and has been found to be effective in the presence of NNRTI resistant mutants, including the signature mutation K103N. ${ }^{20}$ Rilpivirine, like Etravirine, is also a second generation NNRTI and is effective against most NNRTI resistant viruses. The advantage of Rilpivirine is that it comes in a 25 $\mathrm{mg}$ dose and has a longer half half-life (40 hours), making it a useful drug for FDCs. ${ }^{11}$

The integrase inhibitors, which include Raltegravir, the first of its class, and newer agents dolutegravir and elvitegravir, are attractive options for third-line treatment in the developing world as they belong to an entirely new class of drug with no risk of cross-resistance from prior ART exposure in first- and second-line therapy. ${ }^{21}$ They also have a wide range of efficacy for R5 and X4 tropic viruses as well as suppressing the replication of HIV-2. Raltegravir has been found to be able to suppress viral loads to below 50 copies/mL in patients with extensive treatment history. ${ }^{22}$ However, it has a low genetic barrier to resistance which can compromise its efficacy as mutants are able to develop rapidly on a failing regimen. ${ }^{23}$

Additional drugs that can be used to treat HIV resistant strains include CCR5 antagonists and fusion inhibitors, both of which prevent entry of the virus into target cells. Maraviroc, a CCR5 antagonist and the first drug of its class, is currently used in treatment-experienced CCR5-tropic HIV-1 infected patients in high-resource countries. ${ }^{24}$ Recently it has also been shown to have activity against CCR5-tropic HIV-2 infections. ${ }^{25}$ However, its use is severely limited in the developing world on account of the need for HIV tropism testing, which requires advanced laboratory support, prior to its use. Enfuvirtide is the prototype of the fusion inhibitors and was specifically designed for the treatment of HIV-1 infection in ART-experienced adults and children over 6 years of age. It needs to be administered by subcutaneous injection and binds to HIV gp41 protein, thereby inhibiting fusion of HIV with the target cell. For salvage therapy the drug seems to be very valuable in individual cases, although it probably has only a minor role to play in the future of HIV treatment in the developing world. ${ }^{26}$

\section{Dissemination of antiretrovi- rals in developing countries}

The provision of ART across the developing world does not represent a single uniform practice. A number of parallel programmes including those sponsored by national governments, NGOs, as well as private practice all operate in low- and middle-income countries. As the majority of those living with HIV reside in sub-Saharan Africa, and as South Africa has the largest ARV programme in the world, we have chosen to limit our focus to this part of the world. Furthermore, as countries continue to increase their domestic expenditure in funding the HIV/ AIDS response, with South Africa having increased its spending five-fold from 2006-2009 and currently funding more than $75 \%$ of its national roll-out programme, ${ }^{2}$ the authors of this review consider the dissemination of new ARVs in South Africa to be paradigmatic for the rest of the developing world.

The provision of ARVs in the developing world is dependent on multiple factors including political will, regulatory approval, affordability of drugs, as well as procurement and supply management. The global economic crisis that began in 2008 has seen a decrease in donor spending for the HIV/AIDS epidemic in low- and middle-income countries. As twothirds of all AIDS expenditure in Africa comes from external sources, this dependency threatens to destabilize efforts to increase availability of ARVs to where they are needed most, and in particular delay the dissemination of new HIV treatments. The United Nations estimates there is a US $\$ 9$ billion deficit in the HIV funding needed to meet its 2015 goals, and has subsequently proposed a number of strategies that can be adopted to bridge the resource gap. Innovative taxation models such as financial transaction tax, also known as the Robin Hood Tax, is being advocated by UNAIDS and was recently adopted in France where a portion will be put towards the fight against HIV/AIDS. ${ }^{27}$ Furthermore, investing in local manufacturing and simplifying market access to drugs are understood as being central to efforts in ensur- ing sustained access to HIV treatment. UNAIDS recommends countries to take steps towards building local pharmaceutical capacity in order to take full advantage of the flexibilities permitted under the Trade-Related Aspects of Intellectual Property Rights (TRIPS) agreement. $^{2}$

\section{Lowering costs}

Generic drugs have been found to be on average a third of the price when compared with medicines of brand companies. The distribution of these agents in the developing world has been contingent on the lack of product patents in India over older drugs, the issuing of voluntary licenses by brand companies to generic manufacturers, and the nonenforcement of patents. ${ }^{28}$ India is the world's largest producer of generic medicines, accounting for more than $80 \%$ of global ARV purchasing, supplying the majority of ARVs to developing countries. ${ }^{29}$ However, the production and exportation of generic pharmaceutical agents is being challenged by ongoing negotiations for an EU-India Free Trade Agreement that could potentially overrule India's national patent laws. As newer ARVs are more widely-patented than older drugs this threatens the supply of life-saving medicines to where they are needed most. ${ }^{30}$ Drugs that are currently under patent include agents that could be used in third-line regimens which are not currently available to the majority of patients failing second-line treatment in the developing world. These agents include the second generation NNRTI etravarine and PI darunavir (both under patent until 2019), and the first generation integrase-inhibitor raltegravir (under patent until 2022), hence the appeal by activist groups for earlier availability of generic versions of these new medicines. However, as HIV treatment patents have not been universally enforced in the past it is hard to determine whether patent protection will in itself prevent the timely dissemination of costeffective ARVs to the developing world.

Concerns over the quality of generic drugs is being addressed by bioequivalence studies conducted by the FDA, approval of which renders the products eligible for purchase under the PEPFAR programme. Such quality assurance checks are necessary to ensure quality medicines are distributed to developing countries. However, regulatory bodies in the developing world appear to be slowing the distribution of drugs already approved by the FDA. For example, the South Africa Medicines Control Council (MCC) only approved Tenofovir in 2007, whereas it had been approved by the FDA in 2001. Approval of fixed-dose combinations has also proven to be slow. UNAIDS has cited 
weak drug regulatory capacity and inefficient processes as contributing to the lengthy regulatory approval cycle in Africa. The current situation is seen as providing an opportunity for joint ventures and new avenues for collaboration, innovation and technology transfer, with an African Medicines Regulatory Agency being recommended as a possible solution for faster, cheaper and fairer distribution of much needed treatment. ${ }^{27}$ Apart from generics, new drugs are being developed which promise to be much more cost-effective than currently available ones. One such agent is GS-7340, a prodrug of tenofovir. On account of improved pharmacokinetics and cellular penetration, a much lower dose (10-25 mg/daily) is required than what is currently recommended for tenofovir (300 $\mathrm{mg} /$ daily)..$^{11}$ This higher potency at lower concentrations is hoped to translate into significant cost-savings. Another more cost-effective agent is the new heat-stable formulation of atazanavir/ritonavir, which apart from the already mentioned benefit of requiring a single tablet taken daily, is also cheaper than alternatives such as lopinavir/ ritonavir combinations.

\section{Conclusions}

In resource limited settings the most pressing short-term priority in the management of HIV/ AIDS is the scale up of cost-effective antiretroviral therapy. FDCs, which offer reduced pill-burden and hence improved adherence, as well as simplifying prescribing practices and stock and supply management, are likely to play an increasing role in the distribution of ART in the developing world. However, standardizing approaches to drug toxicity will be an ongoing challenge on account of the necessity of HAART to be compatible with a variety of clinical scenarios, including pregnancy, renal disease and coinfection with tuberculosis and hepatitis B \& C. Until newer less toxic combinations are made available it is expected that the roll out of ART in the developing world will be met with increasing adverse drug-reactions that may potentially threaten adherence and the sustainability of such programmes. Furthermore, increased access to ARVs will invariably be met with increased drug-resistance. Thankfully, the vast majority of patients receiving ART in the developing world are still on first-line regimens, thus allowing time for newer agents to be made available as part of third-line treatment options. As we approach the 2015 deadline it is imperative that stakeholders realize the potential role new HIV treatments can have in facilitating attainment of UN targets. That said, the developing world needs access to newly developed ARVs, an AIDS-free generation depends upon it.

\section{References}

1. United Nations General Assembly. Political Declaration on HIV/AIDS: intensifying our efforts to eliminate HIV and AIDS. Resolution adopted by the General Assembly on 10 June 2011. Geneva: UNAIDS; 2011. Available from: http://www.unaids.org/en/media/unaids/co ntentassets/documents/document/2011/0 6/20110610_un_a-res-65-277_en.pdf Accessed: November 2012.

2. UNAIDS. Global Report. UNAIDS Report on the Global AIDS Epidemic 2012. Geneva: UNAIDS; 2012. Available from: http://www.unaids.org/en/media/unaids/co ntentassets/documents/epidemiology/2012/gr2012/20121120_UNAIDS_Global _Report_2012_en.pdf Accessed: November 2012.

3. Seale A, Lazarus JV, Grubb I, et al. HPTN052 and the future of HIV treatment and prevention. Lancet 2011;377:1719.

4. Gilks CF, Crowley S, Ekpini R, et al. The WHO public-health approach to antiretroviral treatment against HIV in resourcelimited settings. Lancet 2006;368:505-10.

5. World Health Organization. Antiretroviral therapy for HIV infection in adults and adolescents: recommendations for a public health approach (2010 revision). Geneva: World Health Organization; 2010. Available from: http://whqlibdoc.who.int/publications/2010/9789241599764_eng.pdf Accessed: November 2012.

6. WHO. Informal consultation on mediumand long-term priorities for ARV drug optimization: moving towards simplification, harmonization and universal access: 29-31 May 2012 Montreux, Switzerland. Geneva: WHO; 2012. Available from: http://apps. who.int/iris/bitstream/10665/75699/1/WHO _HIV_2012.21_eng.pdf Accessed: November 2012

7. Clayden P, Collins S, Daniels C, et al. 2012 Pipeline Report. HIV, HCV and TB, drugs, diagnostics, vaccines, and preventive technologies in development. London: iBase/Treatment Action Group; 2012. Available from: http://i-base.info/wp-content/uploads/2012/07/2012-pipelineweb.pdf Accessed: November 2012.

8. South Africa Info; 2012. Available from: http://www.southafrica.info/about/health/h iv-291112.htm.

9. Semitala F, Kizza HM, Kabungu A, et al. Toxicity of fixed dose nevirapine, lamivudine and stavudine in HIV infected adults. JAIDS 2009;51:1021-5.

10. Ward D, Grant R. Rilpivirine/tenofovir/ emtricitabine fixed-dose combination is an efficacious and well-tolerated switch regimen for patients on therapy. JIAS 2012;15:s18351.

11. Cohen C, Elion R, Ruane $\mathrm{P}$, et al. Randomized, phase 2 evaluation of two single-tablet regimens elvitegravir/cobicistat/emtricitabine/tenofovir disoproxil fumarate versus efavirenz/emtricitabine/ tenofovir disoproxil fumarate for the initial treatment of HIV infection. AIDS 2011;25:F7-12.

12. Dubula-Majola V. PLHIV call on SA government and Wits Reproductive Health and HIV Institute: to phase out D4T and stop the planned low dose D4T study in South Africa. Change.org. Available from: http://www.change.org/petitions/plhiv-callon-sa-governmentand-wits-reproductivehealth-and-hiv-institute-to-phase-out-d4tand-stop-the-planned-lowdose-d4t-studyin-south-africa Accessed: November 2012.

13. Moyle GJ, Sabin CA, Cartledge J, et al. A randomised comparative trial of tenofovir DF or abacavir as replacement for thymidine analogues in persons with lipoatrophy. AIDS 2006;20:2043-50.

14. Southern African HIV Clinicians Society. The 2012 southern African ARV drug resistance testing guidelines. S Afr J HIV Med. 2012;13:162-7.

15. WHO. HIV Resistance Report, 2012. Geneva: WHO; 2012. Available from: h t t p://apps . wh o.int/iris / bi t stream/10665/75183/1/9789241503938_en g.pdf Accessed: November 2012.

16. Marconi VC, Sunpath H, Lu Z, et al. Prevalence of HIV-1 drug resistance after failure of a first highly active antiretroviral therapy regimen in KwaZulu Natal, South Africa. CID 2008;46:1589-97.

17. Nedembi N, Hamers RL, Sigaloff KCE, et al. Transmitted antiretroviral drug resistance among newly HIV-1 diagnosed young individuals in Kampala. AIDS 2011;25:90510.

18. Pujades-Rodriguez M, O'Brien D, Humblet $\mathrm{P}$, et al. Second-line antiretroviral therapy in resource-limited settings: the experience of Medecins Sans Frontieres. AIDS 2008;22:1305-12.

19. Koh Y, Nakata H, Maeda K, et al. Novel non peptidic protease inhibitor with potent activity against multi PI-resistant HIV in vitro. Antimic Ag Chemo 2003;47:3123-9.

20. Andries K, Azijn H, Thielemans T, et al. A novel next generation non nucleoside reverse transcriptase inhibitor active against NNRTI resistant HIV-1. Antimicrob Agents Chemother 2004;48:4680-6.

21. Southern African HIV Clinicians Society. Guidelines for antiretroviral therapy in adults. S Afr J HIV Med 2012;13:114-33.

22. Cooper DA, Steigbeigel RT, Gateli JM, et al. Subgroup and resistance analyses of raltegravir for HIV-1 infection. N Eng J Med 2008;359:355-65. 
23. Grinssztejn B, Nguyen BY, Katlama C, et al. Safety and efficacy of raltegravir in treatment experienced patients with multidrug resistant virus. Lancet 2007;369: 1261-9.

24. Gulick RM, SU Z, Flexner C, et al. Phase 2 study of the safety and efficacy of Maraviroc in treatment experienced HIVinfected patients. JID 2007;196:304-12.

25. Armstrong-James D, Stebbing J, Scourfield A, et al. Clinical outcome in resistant HIV-2 infection treated with raltegravir and maraviroc. Antiviral Res 2010;86:224-6.
26. Lazzarin A, Cltet B, Cooper D, et al. Efficacy of enfuvirtide inpatients infected with drug resistant HIV-1 in Europe and Australia. N Eng J Med 2003;348:2175-85.

27. UNAIDS. AIDS Dependency Crisis: Sourcing African Solutions. Geneva: UNAIDS; 2012. Available from: http://www.unaids.org/en/media/unaids/co ntentassets/documents/unaidspublication/2012/JC2286_Sourcing-AfricanSolutions_en.pdf Accessed: November 2012.

28. Chien CV. HIV/AIDS drugs for Sub-Saharan Africa: how do brand and generic supply compare? PLoS ONE 2007;2:e278.

29. Waning B, Diedrichsen, Moon S. A lifeline to treatment: the role of Indian generic manufacturers in supplying antiretroviral medicines to developing countries. JIAS 2010;13:35.

30. Burrone E, Boulet P, Moon S, et al. ARV patents on the rise? An analysis of ARV patent status in 69 low- and middleincome countries. Washington: XIX International AIDS Conference; 2012. Available from: http://pag.aids2012.org/session.aspx?s=240 Accessed: November 2012. 